\title{
LA PAZ COMO POLÍTICA PÚBLICA Y LA LEGITIMACIÓN DEMOCRÁTICA DE LOS ACUERDOS
}

\section{PEACE AS A PUBLIC POLICY AND THE DEMOCRATIC LEGITIMACY OF AGREEMENTS}

\author{
Natalia Soledad Aprile \\ Universidad del Rosario (Bogotá D.C., Colombia) \\ Mateo Gómez Vásquez \\ Pontificia Universidad Javeriana (Bogotá D.C., Colombia)
}

Recebimento: 17 fev. 2017

Aceitação: 10 jun. 2017

Como citar este artigo / How to cite this article (informe a data atual de acesso / inform the current date of access):

APRILE, Natalia Soledad; VÁSQUEZ, Mateo Gómez. La paz como política pública y la legitimación democrática de los acuerdos. Revista da Faculdade de Direito UFPR, Curitiba, PR, Brasil, v. 62, n. 2, p. 209 - 225, maio/ago. 2017. ISSN 2236-7284. Disponível em: <http://revistas.ufpr.br/direito/article/view/50721>. Acesso em: 28 ago. 2017. DOI: http://dx.doi.org/10.5380/rfdufpr.v62i2.50721.

\section{RESUMEN}

En el presente artículo se analiza el proceso que ha desembocado en la refrendación del Acuerdo de Paz entre el gobierno colombiano y las Farc. A partir de la afirmación de que la paz es una política pública, nos dedicamos a observar cómo se han desarrollado en este caso las distintas etapas que conforman el ciclo teórico de las políticas públicas. Particularmente, nos detenemos a examinar el papel determinante que cumple la ciudadanía, a través del diálogo con las instituciones en la construcción de las políticas públicas y de qué manera ha sido involucrada en las diferentes etapas de este proceso de paz en Colombia. Sostenemos que la participación activa a través de espacios constantes, periódicos e institucionalizados de discusión con todos los sectores de la sociedad es un factor clave para legitimar democráticamente el resultado que pueda arrojar la implementación de este Acuerdo.

\section{PALABRAS CLAVE}

Acuerdo de paz. Política pública. Participación ciudadana. Legitimación democrática.

\begin{abstract}
This article analyzes the process that has led to the endorsement of the Peace Agreement between the Colombian government and the Revolutionary Armed Forces of Colombia (FARC). Based on the assertion that peace is a public policy, we focus on how the different stages that make up the theoretical cycle of public policies have developed in this case. In particular, we examine the determining role of citizenship, through dialogue with institutions in the construction of public policies, and how it has been involved in the different stages of this peace process in Colombia. We hold that active participation through constant, regular and institutionalized spaces of discussion with all sectors of society is a key factor in democratically legitimizing the outcome that may result in the implementation of this agreement.
\end{abstract}




\section{KEYWORDS}

Peace agreement. Public policy. Citizen participation. Democratic legitimacy.

\section{INTRODUCCIÓN}

Los acuerdos de paz tienen las virtudes y las debilidades de un castillo de naipes. Por un lado, son una muestra admirable de un ejercicio de ponderación, balance y precisión, y por el otro, evocan la fragilidad y la inestabilidad a que están expuestos ante cualquier intervención externa. En torno al proceso de paz colombiano y el denodado objetivo de poner fin al conflicto armado interno, corresponde hacer la misma analogía. El 24 de agosto de 2016 se publicó el “Acuerdo Final para la Terminación del Conflicto y la Construcción de una Paz Estable y Duradera”, pactado por las partes negociadoras luego de cinco años de arduas y profundas discusiones. Sin embargo, fue una mayoría estrecha la que, en ejercicio de un mecanismo de democracia directa, se impuso para rechazar la implementación de ese Acuerdo.

El análisis que proponemos en este artículo parte de una consideración muy básica y es que el imperativo de la paz en Colombia depende necesariamente de la consolidación de un proceso deliberativo amplio, leal e incluyente sobre los contenidos y efectos del Acuerdo que se erija como instrumento para la consecución del conflicto. El resultado del plebiscito realizado el 2 de octubre de 2016, pese al sabor amargo que podría dejar con relación a la expectativa de emprender lo más pronto posible un camino seguro hacia la construcción de un país en paz, nos abre un espacio de reflexión interesante y un escenario de debate sugerente para instituir bases más sólidas a partir de las cuales poder levantar los cimientos de un Acuerdo, esta vez edificado en piedra.

En vista de la reverdecida oportunidad y del nuevo Acuerdo Final suscrito el pasado 12 de noviembre, que dice atender "diversas iniciativas de sectores del pueblo colombiano”" proponemos un análisis del proceso de paz en curso desde la categoría de las políticas públicas. Esta perspectiva nos permitirá observar de qué manera se han cristalizado las distintas etapas que conforman el ciclo teórico de las políticas públicas y, sobre todo, hacer foco en el rol que cumplió la ciudadanía en la construcción de esta política de paz. El análisis dogmático que pretendemos realizar tiene como finalidad proponer un enfoque de estudio adicional a un tema generosamente debatido desde varias aristas. Al final del día, la importancia de este ejercicio de reflexión será aprender de la experiencia y contribuir con un modesto aporte en la construcción de la paz colombiana.

\footnotetext{
1 “Acuerdo Final para la terminación del Conflicto y la construcción de una paz estable y duradera”, del 12 de noviembre de 2016, suscrito entre el Gobierno nacional de la República de Colombia y las Farc Ep, disponible en la web: $<$ https://goo.gl/UDMoq8>.
} 


\section{LA PAZ COMO POLÍTICA PÚBLICA}

En el Plan Nacional de Desarrollo que se encuentra actualmente en vigencia, principal instrumento de política pública en Colombia, se ha planteado como objetivo central "construir una Colombia en paz, equitativa y educada, en armonía con los propósitos del Gobierno Nacional, con los estándares de la Organización para la Cooperación y el Desarrollo Económico (OCDE), y con la visión de planificación de largo plazo prevista por la Agenda de Desarrollo post 2015” (artículo $1^{\circ}$ Ley 1753 de 2015). De lo anterior es posible colegir, y las evidencias lo sostienen, que la construcción de paz mediante la superación del conflicto armado es un propósito de Gobierno que reúne las cualidades de una política pública en tanto congrega todos los esfuerzos de la administración por alinear sus ejecutorias en esa ruta. Vale la pena resaltar que en vigencia de la Constitución de 1991 es la primera vez que la construcción de paz se constituye en un pilar y objetivo transversal de la planeación estatal.

A idéntica conclusión arribó la Corte Constitucional en el examen del proyecto de ley estatutaria que reglamentó el plebiscito por la paz, cuando halló plena validez en que el Presidente de la República utilizara este mecanismo de participación ciudadana para la refrendación de lo acordado, toda vez que se trataba de una política pública adelantada por su Gobierno². Incluso, en el recuento jurisprudencial que se hizo en la mencionada sentencia la Corte concluyó que la paz, como un objetivo constitucionalmente imperioso, atribuye "un deber estatal de diseño e implementación de acciones, normativas y de política pública, dirigidas a la superación del conflicto armado y, en general, el logro de la convivencia pacífica”. Quiere decir ello que, dentro del ordenamiento constitucional colombiano, es posible y atinado que se opte por la construcción de una política pública tendiente al logro de la paz.

Ahora bien, una declaración en este sentido implica, en concreto, que el gobierno debe hacer algo para materializar el programa que pretende llevar a cabo (SHAFRITZ; RUSSELL; BORICK, 2015). En una definición más minuciosa y, tal vez, más cercana a la realidad que pretendemos comentar, se ha señalado:

Política pública es un proceso integrador de decisiones, acciones, inacciones, acuerdos e instrumentos, adelantado por autoridades públicas con la participación eventual de los particulares, y encaminado a solucionar o prevenir una situación definida como problemática. La política pública hace parte de un ambiente determinado del cual se nutre y al cual pretende modificar o mantener (GAVILANES, 2010).

\footnotetext{
${ }^{2}$ Corte Constitucional, Sentencia C-379 de 2016.
} 
Bajo este marco, no puede desconocerse la conexión indisoluble de análisis que puede hacerse entre el proceso que condujo a la definición del Acuerdo de Paz en Colombia y la teoría de las políticas públicas. Precisamente, el análisis de las políticas públicas constituye una corriente o metodología específica dentro de la Ciencia de la Administración, que propone focalizar la perspectiva de estudio sobre las acciones del gobierno y, con esto, abordar de manera dinámica el objeto de la Administración Pública. Por lo anterior es usual referirse a las políticas públicas como un proceso o ciclo con diferentes etapas (FERRARO, 2009).

El ciclo de las políticas públicas está conformado por cuatro momentos analíticos, que es útil desligar a efectos de su estudio, pero que no obedecen a un criterio temporal, puesto que pueden no darse en el orden señalado o, incluso, confluir en determinados momentos. Así, siguiendo el esquema descrito por Shafritz, Russell \& Borick, el proceso se compone de las siguientes etapas: (i) la formación de la agenda; (ii) las decisiones (o no decisiones) de políticas; (iii) la implementación de un nuevo programa o la modificación de una política pública existente y (iv) la evaluación crítica y las evaluaciones formales.

Todas estas fases forman un flujo que integran el curso de acción dirigido a definir las políticas públicas de un Estado y que están permeadas por un feedback constante e intermitente que permite incluir nuevos temas en la agenda o replantear aquellos que ya fueron incorporados al proceso. A su vez, como recuerdan los autores citados, todo en este ciclo está decididamente influenciado por el entorno político (SHAFRITZ; RUSSELL; BORICK, 2015). Dicho lo anterior, y habiendo definido prima facie que la construcción de paz es una política pública, resulta interesante analizar cómo sucedieron estas fases que han sido categorizadas por la doctrina.

\subsection{LA PAZ COMO TEMA DE LA AGENDA DE LAS POLÍTICAS PÚBLICAS}

En cuanto a la formación de la agenda podemos encontrar que, dadas las características esenciales de los procesos de paz, cuyo propósito es poner de acuerdo a dos contendores políticos y militares, esta fase, además de esencial, se debe llevar a cabo con el mayor hermetismo y celo posible (FISAS, 2010). La negociación entre el Gobierno y las Farc no fue diferente pues respondió a la necesidad de mantener en secreto la decisión de encarar una agenda de acuerdos. Después de una serie de acercamientos informales para medir la voluntad de negociación de cada una de las partes y, concretamente, de algunos encuentros exploratorios llevados a cabo en La Habana entre el 23 y el 26 de agosto de 2012, las partes lograron sentar seis puntos como ejes esenciales para la consecución de un acuerdo para poner fin al conflicto armado que los enfrenta. Tales puntos, como es de público 
conocimiento, fueron la política de desarrollo agrario integral, la participación en política, el fin del conflicto, la solución al problema de las drogas ilícitas, la reparación de las víctimas, y lo relacionado con implementación, verificación y refrendación.

Es decir, las Farc y el Gobierno nacional sellaron una agenda compartida sobre la cual trabajarían para construir un acuerdo tendiente a la construcción de la paz. Ahora bien, dicha agenda tuvo como característica principal la de no ser una hoja de ruta para simplemente callar los fusiles, sino además para generar condiciones de convivencia pacífica y erradicar las causas del conflicto. Esto significa que se construyó sobre un concepto de paz positiva que, por definición, busca un alcance mayor a la simple cesación de las hostilidades (GALTUNG, 1969). En suma, la formación de la agenda para el caso de la política pública de paz ha tenido como embrión el consenso alcanzado por dos partes enfrentadas en un conflicto armado, que hallaron en el abordaje de un itinerario de asuntos sustantivos, la fórmula para construir un acuerdo para finalizar el conflicto y emprender una ruta de construcción de paz.

En este escenario, es preciso reflexionar sobre el lugar que ocupó la ciudadanía para influir en la definición de la agenda. En la etapa de formación de la agenda de las políticas públicas se difunden las ideas o asuntos por medio de diversos canales de comunicación, hasta terminar siendo considerados por una institución pública, como pueden ser una legislatura, el poder ejecutivo, una agencia independiente de gobierno o un tribunal. Es interesante resaltar que, desde esta óptica, la formación de la agenda no está restringida a los políticos, sino que muchas personas pueden participar, existiendo determinados grupos, llamados agenda setters, que tienden a tener mayor influencia y ejercerla con mayor frecuencia (FERRARO, 2009).

La participación de los ciudadanos y sus organizaciones para generar las políticas públicas “desde abajo” (CANTO CHAC, 2008) tiene la virtud de hacer visibles ciertos temas, ampliar los derechos, generar sentimientos de pertenencia social y garantizar el derecho a una buena administración ${ }^{3}$. Esta modalidad responde a la reconfiguración que se ha dado en las relaciones entre Estado y sociedad, que constata que el Estado ha dejado de ser el centro y eje del poder público y la sociedad le ha ganado protagonismo en la detentación de ese poder (ESTEVE PARDO, 2013). Atrás quedaron los modelos lineales de política pública, según los cuales ésta se transmite desde el gobierno como oferta hacia la sociedad para que ésta la acepte como ordenación de sus intereses. Por ello se

\footnotetext{
${ }^{3}$ Este derecho, reconocido expresamente en la Carta Europea de Derechos Humanos, se concibe como un conjunto de valores a través de los cuales la ciudadanía juzga hoy la legitimidad de las administraciones públicas (CERRILLO; MARTÍNEZ, 2005). Según se afirma, "La buena administración está muy relacionada con la autoexigencia administrativa de calidad en los servicios públicos, aunque va más allá de ella, pues aporta un elemento externo configurado por los ciudadanos que ejercen un derecho y presionan para el impulso de dicha calidad” (PRATS, 2010).
} 
propone "trascender una noción relacionada puramente con la delegación y la representación de la democracia, en favor de canales múltiples de consulta, participación, diálogo y concertación social [...]” (CEPAL, 2007). En concreto, esto significa que la ciudadanía se encuentra indudablemente implicada en la articulación de las políticas, utilizando los mecanismos que proporciona el sistema democrático y exigiéndole al Estado que evacúe sus demandas.

En el marco del análisis propuesto, podríamos aseverar que esta etapa de formación de la agendafue mediada por instituciones que le daban garantías a las partes como la Universidad Nacional a través del Centro de Pensamiento y Seguimiento al Proceso de Paz y el sistema de Naciones Unidas en Colombia. Precisamente a ellos se les encargó, por parte de la mesa negociadora, la realización de una serie de foros regionales y nacionales para abordar las principales temáticas de la agenda de paz ${ }^{4}$. En esta misma fase se identifica la puesta a disposición de la ciudadanía de una herramienta virtual para el envío de propuestas relacionadas con la agenda de negociación, la cual hasta la fecha ha canalizado $16820^{5}$.

Por otra parte, la cobertura mediática del proceso ha tenido una influencia poderosa en la instalación del tema en la sociedad y asimismo en la percepción social del asunto. La teoría de la agenda setting se ocupa, precisamente, de estas dos dimensiones de influencia (MCCOMBS y EVATT, 1995) y es claro que la prensa tradicional, como la radio, los periódicos o la televisión, así como los medios que han surgido con el avance tecnológico a través de internet y la telefonía inteligente, son responsables del posicionamiento de los temas en la agenda pública y de difundir las labores que se adelantan a favor de la paz. No desconocemos que la información es un elemento muy valioso en los procesos de construcción de paz, pues el éxito de los mismos depende en gran medida de la capacidad para transmitir la realidad del conflicto que, para algunos, se presenta en un contexto alejado de su propia realidad. Pero tampoco es posible soslayar el hecho concreto de que la sociedad civil no participó directamente, a través de la deliberación y discusión colectiva, en la fijación de la agenda. Incluso se puede colegir que el revés plebiscitario del 2 de octubre, así como los obstáculos que se deberán afrontar en la implementación programática del acuerdo de paz, se encuentran fundados en este déficit de participación. No obstante tampoco se puede obviar que la construcción

\footnotetext{
${ }^{4}$ Informe preliminar Foro Nacional de Víctimas, disponible en web: <https://goo.gl/BB3LWd>; Informe y Balance General Foro de Desarrollo Agrario Integral (enfoque territorial), disponible en web: <https://goo.gl/F2DRVf >; Informe y Balance General Foro Participación Política, disponible en web: <https://goo.gl/uJLyRv>; Informe y Balance General Foro Nacional y Regional sobre la solución al problema de las drogas ilícitas, disponible en la web: $<$ https://goo.gl/pZs6RZ>.

${ }^{5}$ Esta herramienta se encuentra contenida en la página web: <https://goo.gl/kvQokk>. En el siguiente vínculo se encuentra la relación de las propuestas: <https://goo.gl/kvQokk>.
} 
bilateral y consensuada de la agenda de conversaciones es la principal medida de confianza entre dos partes inmersas en un conflicto armado.

\subsection{LA DECISIÓN DE LA POLÍTICA PÚBLICA DE PAZ}

La segunda etapa identificada en el esquema propuesto por Shafritz, Russell \& Borick es la del momento decisorio de la política pública. Es decir, aquel donde se define el curso a seguir de conformidad con la agenda planteada para el logro del objetivo previsto; para el caso que nos ocupa, la construcción de paz. Conscientes de la carencia democrática de los procesos de negociación, pues estos involucran en su dinámica exclusivamente a las partes en conflicto, el Gobierno y las Farc acordaron que en la mesa de negociación se diseñarían las líneas gruesas de desarrollo de la agenda, pero que, en todo caso, ello debía ser adoptado mediante los mecanismos contemplados por la Constitución de 1991. Lo anterior se traduce en que el Acuerdo Final, en sus contenidos sustantivos y procedimentales, si bien serían formulados en la mesa de negociación, su operatividad en el ordenamiento jurídico interno dependería del ejercicio de las competencias atribuidas por la Constitución en cada materia al órgano legislativo y al ejecutivo, siempre y cuando el poder electoral refrendara lo acordado. Ello se deduce, además del contenido del Acto Legislativo para la paz (01 de 2016), del acuerdo del 12 de mayo de 2016 alcanzado por las partes ${ }^{6}$.

Dejando de lado por el momento los aspectos formales que rodearon las negociaciones y el Acuerdo alcanzado, no es posible eludir en esta etapa de análisis que los actores que lideran el proceso de paz en Colombia representan, sin lugar a dudas, diversos intereses e ideologías. Lo que se traduce en una pluralidad de soluciones posibles para tratar o resolver el conflicto. Además de las autoridades públicas implicadas hay actores políticos, organizaciones intermedias, entre las que se cuentan organizaciones de la sociedad civil, sindicatos y movimientos sociales, y además de una porción importante de la sociedad que clama verse reflejada y reconocida en los programas y cursos de acción a seguir. Esta multiplicidad de intereses y actores explica por qué las decisiones no son siempre las más racionales o las más coherentes; pues son el resultado de la confrontación y negociación. Pero justamente la riqueza de estos procesos deliberativos radica en que su resultado emana de una pluralidad de discursos a partir de los cuales se establece una voluntad común.

El Acuerdo alcanzado por el gobierno y las Farc fue el producto de un largo y complejo proceso que poco tiene que ver con el esquema clásico de la perspectiva de la decisión racional,

\footnotetext{
${ }^{6}$ Comunicado Conjunto \#69. La Habana, Cuba, 12 de mayo de 2016, disponible en la web: <https://goo.gl/kvQokk>.
} 
propuesta originalmente por Laswell para explicar el proceso común a toda decisión de política pública adoptada en forma racional (LASSWELL, 1956). Por el contrario, subyace en este proceso la teoría crítica de esa perspectiva lineal que propone que las alternativas de solución posibles compiten en una clara concepción pluralista que predomina en las relaciones en los procesos de toma de decisiones y de la distribución del poder en la sociedad. Este modelo, llamado de perspectiva incremental, explica de un modo más realista que las decisiones de política pública son medidas por el autointerés. Es decir, son mediadas por la lucha entre actores políticos que actúan en defensa de sus propios intereses o ideologías y ganan terreno en la medida en que se van imponiendo unas sobre otras (LINDBLOM, 1959).

De acuerdo con el planteamiento de la visión incremental, como teoría que busca explicar los mecanismos de producción de las decisiones de políticas públicas, los diferentes grupos tienen capacidad para defender sus intereses y para que ninguno sea ignorado, resolviéndose el conflicto a través del proceso de ajuste mutuo (PORTA, 1988). Si bien esta teoría ha sido objeto de críticas, vinculadas por ejemplo con la existencia de una desigual distribución del poder que impide esa dinámica de conciliación equitativa (SUBIRATS, 2001), lo cierto es que hay algo innegable en el planteamiento y es que la formación e implementación de una política pública es, inevitablemente, el resultado de la interacción entre actores diversos, con intereses, metas y estrategias disímiles. En el caso, el proceso que condujo a la formulación del Acuerdo Final en su primera versión respondió a la lógica de la negociación y el debate, en el que pugnaron las partes intervinientes hasta alcanzar un nivel óptimo de satisfacción que se sometió al escrutinio de la ciudadanía.

Sin embargo, es posible precisar que en esa etapa decisoria, inicialmente, no se promovió con intensidad como un diálogo inclusivo de todos los potencialmente afectados, pues la decisión cerrada desde el Ejecutivo fue sometida a consulta pública en la recta final. Recién, luego de haber sido rechazado ese Acuerdo por la mayoría de los ciudadanos que confluyeron en las urnas, se consuma en un nuevo Acuerdo la participación de los sectores de la sociedad y se anuncia que éste será sometido a un procedimiento de refrendación, mediante sistemas de participación ciudadana que no se limitan al plebiscito ${ }^{7}$. El devenir de los acontecimientos nos demostrará si el nuevo Acuerdo Final se arropará con ciertos valores democráticos que el anterior no incluía para reforzar su legitimidad política.

\footnotetext{
7 “Acuerdo Final para la terminación del Conflicto y la construcción de una paz estable y duradera” del 12 de noviembre de 2016, suscrito entre el Gobierno nacional de la República de Colombia y las Farc Ep, disponible en la web: $<$ https://goo.gl/UDMoq8>.
} 


\subsection{LA PUESTA EN PRÁCTICA Y LA EVALUACIÓN DEL ACUERDO DE PAZ}

Cuando hablamos de la evaluación de las políticas públicas, no nos estamos refiriendo únicamente a la fiscalización que podría hacerse en la etapa última de su implementación para verificar los resultados de la ejecución. En rigor, también aludimos a la valoración que debe realizarse a lo largo de todo el ciclo, desde el mismo diseño y elaboración de la política pública. En este sentido, se entiende que la evaluación consiste en "aportar elementos al proceso de toma de decisiones (reformulación), que permitan mejorar los efectos de la actividad evaluada y contribuyan a la rendición de cuentas y a la generación de ciudadanía” (CARDOZO BRUM, 2013). La evaluación equivale, en esos términos, a una instancia para reflexionar sobre el camino adoptado, monitorear el impacto que puede tener la política pública decidida y proponer ajustes o cambios. Habida cuenta de que el ejercicio de evaluación debe realizarse durante todo el proceso (SUBIRATS, 2001), se torna necesario aprender de la experiencia que ha dejado el resultado del plebiscito celebrado el 2 de octubre, en la tarea de encontrar caminos que permitan reencauzar el proceso de paz colombiano.

Desde la disciplina de estudio de la Administración Pública se han propuesto varios enfoques que representan modelos de implementación de políticas públicas (FERRARO, 2009). Los más recientes involucran como aspecto clave la negociación entre actores y hacen énfasis en modelos deliberativos y participativos, respondiendo así a los postulados de las reformas administrativas afines al enfoque de la gobernanza (CANTO CHAC, 2008). Estos modelos “fundados en la recuperación de la confianza y legitimidad de la acción pública, y buscando contar con la ciudadanía como centro y protagonista de su propio desarrollo"8, se alejan del esquema tradicional conforme al cual las decisiones de políticas públicas emanan jerárquicamente del Estado como centro del poder político ${ }^{9}$.

En esta visión, la arquitectura estatal exige hoy en día una mayor interacción entre Estado y sociedad en la ejecución de las políticas públicas. Precisamente, esta garantía de participación se contempla en el Acuerdo Final, tanto en su versión original como en la nueva, al proclamar que "La participación ciudadana es el fundamento de todos los acuerdos que constituyen el Acuerdo Final.

\footnotetext{
${ }^{8}$ Preámbulo, Carta Iberoamericana de Gobierno Abierto, Aprobada por la XVII Conferencia Iberoamericana de Ministras y Ministros de Administración Pública y Reforma del Estado, Bogotá, Colombia, 7 y 8 de julio de 2016.

${ }^{9}$ Más allá de la lectura crítica que podría hacerse del concepto de gobernanza, lo cierto es que hay un consenso básico respecto de que el modelo representa un cambio en el sentido y en el proceso de gobernar que desplaza del centro de la escena al gobierno. En efecto, no podemos eludir que cuando se habla de participación ciudadana en el marco de las políticas públicas, se aborda indefectiblemente el asunto de la gobernanza que, como es sabido, logró amplia difusión cuando el Banco Mundial y otros organismos internacionales promotores de políticas de desarrollo anunciaron la llamada “crisis in governance”. En palabras de Mayntz, "actualmente se recurre a governance sobre todo para indicar un nuevo estilo de gobierno, distinto del modelo del control jerárquico y caracterizado por un mayor grado de cooperación y por la interacción entre el Estado y los actores no estatales al interior de redes decisionales mixtas entre lo público y lo privado” (MAYNTZ, 2000).
} 
Participación en general de la sociedad en la construcción de la paz y participación en particular en la planeación, la ejecución y el seguimiento a los planes y programas en los territorios, que es además una garantía de transparencia” ${ }^{10}$. Por añadidura, el tema de la participación ciudadana se hace presente en varios pasajes del Acuerdo adquiriendo un protagonismo destacable en el texto.

De manera pues que podríamos afirmar que, al momento de la implementación de este Acuerdo, existirán muchas herramientas que pondrán a prueba la capacidad institucional y la madurez de la sociedad para articular los espacios de diálogo y deliberación que están contemplados. Ahora bien, en el análisis aquí planteado, la intervención de la ciudadanía no se aprovechó en la etapa de diálogos exploratorios para construir la agenda de paz ni tampoco en la definición de los puntos centrales del Acuerdo. Más allá de una plataforma virtual en la que los ciudadanos podían realizar propuestas, evidentemente la realidad demostró que era necesario una discusión e interlocución más profunda con los sectores de la sociedad civil, para enriquecer las negociaciones de la mesa y robustecer la legitimidad de los acuerdos.

Y también ha evidenciado la realidad que el mecanismo del plebiscito, al limitar la definición de una política de semejante trascendencia a una decisión binaria, no convenció a la ciudadanía para inclinarse mayoritariamente a aceptar el Acuerdo. Esta circunstancia, desde el punto de vista del ciclo de las políticas públicas, plantea un escenario de necesaria retroalimentación o feedback que conduce a replantear el camino seguido. Este reto ha sido encarado desde el triunfo del No en el plebiscito, con la premura que exige no perder el impulso negociador de las partes, a través del intercambio de argumentos con sectores que se consideraban aplazados, con el fin de construir colectivamente un Acuerdo más integrador que contemple los ajustes y precisiones que estos sectores reclamaron.

Por lo visto hasta aquí, el análisis teórico del ciclo de las políticas públicas nos permite visualizar que la participación ciudadana es vital en todas las etapas de esta clase de procesos ${ }^{11}$. Al respecto, en el Informe de Desarrollo Humano: “Callejón con Salida” del PNUD para Colombia de 2003, se advertía que el Estado debía mostrarse más abierto y menos desconfiado en las iniciativas de la sociedad civil y que, a su turno, la sociedad civil no debía olvidar “que es un poder, que el poder

\footnotetext{
10 “Acuerdo Final para la terminación del Conflicto y la construcción de una paz estable y duradera” del 12 de noviembre de 2016, suscrito entre el Gobierno nacional de la República de Colombia y las Farc Ep, disponible en la web: $<$ https://goo.gl/UDMoq8>, páginas 6 y 7.

${ }^{11}$ Incluso más, cuando hoy se habla de gobierno abierto, se hace referencia a que uno de sus pilares es "Fortalecer los espacios de participación ciudadana en los asuntos públicos y en la toma de decisiones que les atañen, promoviendo además la colaboración en la búsqueda e implementación de soluciones en un esquema de mayor responsabilidad compartida que pueda aprovechar las capacidades distribuidas y la inteligencia colectiva de los actores sociales” (NASER; ALUJAS, 2014).
} 
existe para ser usado y que usarlo bien es jugarse a la paz"12. Cabe insistir en que la participación no es sólo predicable respecto de momentos puntuales en la toma de decisiones sino que el ideal democrático exige que la ciudadanía tome su lugar a lo largo de todo el ciclo. Lo que no significa que se prescinda de la institucionalidad pública, pues en esta visión la sociedad civil carece de autoridad para tomar por su cuenta decisiones de política pública, sino que su tarea puede consistir en proveer información, ejercer presión e influir activamente en el diagnóstico, la programación, la ejecución y la evaluación de las políticas públicas (VALENCIA AGUDELO, 2012).

\section{LA LEGITIMACIÓN DEMOCRÁTICA DE LA POLÍTICA PÚBLICA DE PAZ}

Mucho se ha escrito sobre la participación ciudadana en los últimos años, incluso por parte de quienes cuestionan seriamente su funcionamiento ${ }^{13}$. En esta oportunidad, nos interesa dejar trazadas sólo unas líneas generales que servirán de marco para observar el funcionamiento que ha tenido en la práctica el mecanismo de refrendación utilizado para legitimar el Acuerdo de Paz. Por ello, partimos de la base de que, si bien el fenómeno participativo es discutible, por las flaquezas que se demuestran en el plano empírico y que, podríamos arriesgarnos a asegurar, son congénitas a todo espacio de poder en que puedan desarrollarse, los diagnósticos negativos no deben ser motivo de resignación. En todo caso, la balanza debe siempre inclinarse a favor de estas prácticas porque al menos acercan las preferencias de los ciudadanos a los representantes, fortaleciendo el vínculo de confianza entre ambos.

Existe consenso acerca de que el fenómeno de la participación de la ciudadanía en la gestión pública ha sido el producto de un nuevo paradigma que ha desembocado en la resignificación del papel del Estado y de la sociedad en la construcción de las políticas públicas. En el proceso que analizamos, tanto en la construcción como en la reconstrucción del Acuerdo Final, que será la plataforma sobre la cual se construya el proceso de paz en adelante, queda en evidencia que el ejercicio del diálogo es el motor que legitimará la política a implementarse. Ahora bien, en este diseño político-institucional que tiene por objeto que los ciudadanos se hagan parte de las decisiones públicas fundamentales que son de su interés, exponiendo sus preferencias y las razones que justifican su

\footnotetext{
12 PNUD. Informe Nacional de Desarrollo Humano para Colombia 2003: "El conflicto, callejón con salida”. Bogotá: Panamericana, p. 459.

${ }^{13}$ Existen estudios críticos sobre la efectividad real de los mecanismos de participación ciudadana que hacen eje en la falta de virtualidad de la sociedad civil para la provisión del bienestar general (ARCIDIACONO, 2011) y en la falta de integración de los diversos actores de la sociedad civil como para propender a una efectiva rendición de cuentas (FOX, 2006).
} 
opción, se propone una mejora institucional al modelo de la democracia participativa, asentada en el pensamiento republicano de Rousseau (ROUSSEAU, 1979).

Bajo esta óptica, la democracia participativa adquiere dos variantes. Una es la forma directa, defendida por Rousseau, que se caracteriza por la introducción de mecanismos institucionales que garanticen la participación de la ciudadanía en la vida pública, a través de instrumentos como los referéndums, la iniciativa popular de ley, la revocatoria del mandato, entre otros. La otra es la forma deliberativa de la regulación democrática participativa, sustentada en la teoría comunicativa de Habermas, que se define a partir del contenido del proceso decisional, el cual, con el propósito de lograr decisiones justificadas, integra en un diálogo inclusivo a todas las partes afectadas por la decisión que, ante un desacuerdo, se debe tomar (GARGARELLA, 2013). Así, en un intercambio de razones y argumentos, tanto ciudadanos como representantes construyen cooperativamente y de manera consensuada la decisión tendiente a la resolución del desacuerdo.

Esta fórmula deliberativa de la democracia se presenta como el ideal regulativo ${ }^{14}$ en razón de la imparcialidad de su resultado. O, dicho en otros términos, como lo explica Nino al referirse al valor epistémico de la democracia:

[...] la concepción deliberativa de la democracia ve a esta última como profundamente interrelacionada con la moral y se apoya sobre su poder para transformar preferencias moralmente aceptables [...] el consenso alcanzado después de un ejercicio de discusión colectiva debe ser de algún modo confiable en el proceso de conocimiento de asuntos morales (NINO, 1997).

En esta vertiente deliberativa, la democracia se traduce en una herramienta procedimental que permite a los intervinientes del debate público exponer sus puntos de vista con el propósito de persuadir a sus interlocutores pero, al mismo tiempo, les da la oportunidad de atender a estos últimos para permitirles ser persuadidos ante los argumentos del resto de intervinientes.

Holmes, con mayor precisión, apunta que en esta concepción la democracia es el "gobierno por discusión pública” y no sólo la imposición de la voluntad mayoritaria. Así, afirma que será

\footnotetext{
${ }^{14}$ Ver entre otros: Bruce Ackerman, La política del diálogo liberal (1998); Ronald Dworkin, Los derechos en serio (1989); Owen Fiss, Between Supremacy and Exclusivity (2006); Roberto Gargarella, El nuevo constitucionalismo dialógico frente al sistema de los frenos y los contrapesos (2013), El ideal de la democracia deliberativa en el análisis del sistema representativo. Algunas notas teóricas y una mirada sobre el caso de la Argentina (1995), Los fundamentos legales de la desigualdad: el constitucionalismo en América (1776-1860) (2005), La justicia frente al gobierno. Sobre el carácter contramayoritario del poder judicial (1996); Stephen Holmes, El precompromiso y la paradoja de la democracia (1999); Robert Post y Reva Siegel, Constitucionalismo Democrático. Por una reconciliación entre Constitución y pueblo (2013); Rodrigo Uprimny, La judicialización de la política en Colombia: casos, potencialidades y riesgos (2007); Joshua Cohen, Deliberation and democratic legitimacy (2003); Carlos F. Rosenkrantz, La teoría episistémica de la democracia revisitada (2004).
} 
democrática la decisión que haya tenido ocasión de ser discutida a profundidad contemplando todos los puntos de vista posibles incluyendo, especialmente, a los más críticos. En tal sentido, un diseño institucional que profundice la deliberación incluyente y participativa, garantizaría en su criterio que la adopción de decisiones de gobierno provenga de debate ricos y con información completa y, consecuentemente, desprovista de arbitrariedades. De manera pues que la deliberación no es solo una garantía para proteger a la minoría sino también un instrumento para asegurar que la mayoría corrija e instruya su decisión (HOLMES, 1999).

En el marco señalado, podemos observar que en la aprobación e implementación de la política de paz en el caso colombiano, el mecanismo de democracia participativa directa ha tenido que ser complementado con un ejercicio de democracia deliberativa. En efecto, luego de la realización del plebiscito, en un claro contexto de feedback, los sectores de la sociedad que se consideraron apartados del debate o que no encontraron suficiente representación en el Acuerdo, exigieron al gobierno que se prolongara la discusión y les concediera la oportunidad de expresar sus intereses para poder aceptar lo que se estaba proponiendo desde la institucionalidad. Esto demuestra que, si bien es válida la adopción de cualquier sistema derivado de uno u otro modelo de democracia participativa, la poca discusión previa y la simplificación de un sistema que permite votar por sí o por no, en el caso de una decisión tan relevante el mecanismo del plebiscito quedó pequeño. Lo anterior no quiere decir que la opción de refrendar el Acuerdo Final mediante un mecanismo de participación directa como el plebiscito haya sido desatinada. Lo que parece haber sido un error es que el proceso en sí mismo no haya estado acompañado de espacios constantes, periódicos e institucionalizados de discusión con todos los sectores de la sociedad, especialmente los más críticos del Acuerdo.

Indudablemente, son las condiciones de tiempo, modo y lugar de cada sociedad las que aconsejan la adopción de uno u otro modelo de democracia participativa y la profundización en una u otra concepción de regulación democrática. Tampoco puede negarse que, al final, la deliberación y el intercambio argumentativo entre los ciudadanos y las instituciones contribuyen a reforzar el ideal democrático, pues el diálogo institucional, por sí solo, a veces no alcanza a efectos de la democratización de la deliberación pues excluye de la dinámica dialógica a los ciudadanos que son, precisamente, quienes legitiman la resolución de los desacuerdos en una sociedad.

Por tal motivo, el poder potencial que tienen los ciudadanos al participar a través del diálogo en la construcción del acuerdo de paz será determinante para legitimar democráticamente el resultado que pueda arrojar su implementación. Que la ciudadanía tenga la posibilidad de persuadir y ser persuadida sobre una lectura específica del acuerdo, es lo que subsana el déficit democrático que podría atribuirse a una política pública impuesta por los poderes públicos. Tal consideración nos pone 
de presente que una decisión legitimada democráticamente no sólo depende de quién propone sino de quien tiene la posibilidad de hacer oír su voz.

Los hechos indican que, dada la fragilidad del cese al fuego de hostilidades bilateral y definitivo por cuenta del resultado plebiscitario, la refrendación del Acuerdo Final con sus modificaciones se hará mediante el Congreso de la República a través del trámite de una iniciativa legislativa. Ello, por supuesto, volvería más estrecho los escenarios de deliberación democrática, plural, abierta e incluyente. No obstante, el reto que se avecina para la sociedad y el Estado colombiano es el de imbuirse en un diálogo sincero, amplio y leal, sobre todos y cada uno de los puntos sobre los cuales las Farc y el Gobierno se han puesto de acuerdo para callar los fusiles pero, especialmente, eliminar las condiciones por las cuales el conflicto emergió y perduró por tantos años.

\section{CONCLUSIONES}

Con base en lo anterior podemos colegir, con meridiana certeza, que el rol que adquiere el control crítico ciudadano en el ciclo de las políticas públicas es un elemento crucial, de acuerdo con los estudios que proponen la democratización de la gestión pública ${ }^{15}$. Y a nuestro juicio, este es el elemento que resulta más interesante de analizar en la política pública de paz que se pretende implementar en Colombia. Como observadores del proceso, podemos advertir que el involucramiento de la ciudadanía ha pasado por al menos tres fases: una primera, de absoluto distanciamiento signado por el hermetismo de la mesa de negociación, bien por encontrarse el proceso en etapa exploratoria, o bien porque los primeros meses de negociación las partes estaban concentradas en consolidar la confianza que requería para emprender la etapa formal de negociación. Una segunda etapa, donde la participación ciudadana fue agenciada por instituciones que les daban garantías a las partes como la Universidad Nacional a través del Centro de Pensamiento y Seguimiento al Proceso de Paz y el sistema de Naciones Unidas en Colombia. Y, finalmente, se puede identificar una tercera etapa de pleno involucramiento de la sociedad, que está signado por la definición del plebiscito como mecanismo de refrendación. A partir de ese momento, y en la etapa posterior a la jornada plebiscitaria, es posible evidenciar una presencia activa de los más diversos sectores políticos, económicos y

\footnotetext{
${ }^{15}$ Con relación a este tema, podemos citar los trabajos de Cunill Grau, N. (1995). La rearticulación de las relaciones Estado-sociedad: en búsqueda de nuevos sentidos. Revista del CLAD Reforma y democracia, (4), 25-58; Cohen, J. y A. Arato (1995). Civil Society and Political Theory. Cambridge: MIT Press. Más cerca en el tiempo, Subirats, J. (2011). Otra sociedad. ¿Otra política? Del “no nos representan” a la democracia de lo común. Barcelona: Icaria; Parsons, W. (2013). Políticas públicas: una introducción a la teoría y la práctica del análisis de políticas públicas. FLACSO México.
} 
sociales del país en el curso del proceso de paz, que es el curso de la política pública al final de cuentas.

Con ello quisiéramos intentar esbozar las siguientes reflexiones:

- La resolución de un conflicto armado por la vía dialogada demanda de la administración pública comprometida con ese objetivo desplegar una estrategia enmarcada en la construcción y puesta en marcha de una política pública, con las sucesivas fases que ello comprende.

- $\quad$ Dicho proceso debe partir del supuesto de que los intereses y puntos de partida de cada uno de los actores son distantes, antagónicos y enfrentados. Asimismo, en aquellos procesos de terminación del conflicto que se construyen bajo el parangón de un concepto de paz positiva, debe considerarse que hay sectores de la sociedad cuyas posturas sobre los medios para erradicar las causas del conflicto pueden ser igualmente distantes a los de las partes en la mesa de conversación.

- $\quad$ En la medida en que en estos procesos de paz se adoptan medidas que afectarán en adelante la vida de la Nación, es absolutamente imprescindible un involucramiento profundo de todos los sectores sociales, especialmente de los contradictores del proceso, para que el fin del conflicto se enmarque en un escenario de legitimidad política.

- $\quad$ La democracia directa, aunque deseable e imperiosa constitucionalmente, puede ser limitada para dotar de legitimidad política un proceso de paz. La legitimidad reposa, ciertamente, en que en las etapas previas a la refrendación del acuerdo todos los sectores sociales, pero especialmente los contradictores, tengas espacios amplios, plurales e institucionalizados para transmitir sus reclamos. Claro, lo anterior bajo el supuesto de que todas las partes y especialmente los contradictores ejercen el derecho a la participación de manera leal, es decir, con el propósito de persuadir a los formuladores de la política pública de paz, pero a su vez con la disposición de dejarse persuadir.

\section{REFERENCIAS}

ARCIDIACONO, Pilar. «El protagonismo de la sociedad civil en las políticas públicas: entre el “deber ser” de la participación y la necesidad política». Revista del CLAD Reforma y Democracia No 51, 2011.

BEN-DOR, Oren. Constitutional limits and the public sphere. Oxford: Hart Publishing, 2000.

BICKEL, Alexander M. The Least Dangerous Branch: The Supreme Court at the Bar of Politics. New York: Yale University Press, 1986.

BROUCHOUD, María Fernanda Martínez. «Gobernanza y legitimidad democrática». Reflexión Política, 2010: 96-107. 
CANTO CHAC, Manuel. «Gobernanza y participación ciudadana en las políticas públicas frente al reto del desarrollo». Política y cultura, nº 30 (2008): 9-37.

CARDOZO BRUM, Myriam. «De la evaluación a la reformulación de políticas públicas». Política y cultura, 2013: 123-149.

CEPAL. «Cohesión social: inclusión y sentido de pertenencia en América Latina y el Caribe». Santiago de Chile, 2007.

CERRILLO I MARTÍNEZ, Agustí. La gobernanza hoy: 10 textos de referencia. Madrid: Instituto Nacional de Administración Pública, 2005.

EPP, Charles. La revolución de los derechos. Abogados, activistas y cortes supremas en perspectivas comparadas. Buenos Aires: Siglo XXI, 2013.

ESTEVE PARDO, José. La nueva relación entre Estado y sociedad. Madrid: Marcial Pons, 2013.

FERRARO, Agustín. Reinventando el Estado. Por una administración pública democrática y profesional en Iberoamérica. Madrid: Instituto Nacional de Administración Pública, 2009.

FISAS, Vincenç. «Introducción a los Procesos de Paz». Quaderns de Construció de Pau, Febrero 2010: 5-22.

FOX, Jonathan. «Sociedad civil y políticas de rendición de cuentas». Perfiles Latinoamericanos. Revista de la Facultad Latinoamericana de Ciencias Sociales, 2006: 33-68.

GALTUNG, Johan. «Violence, Peace, and Peace Research». Journal of Peace Research, 1969: 167191.

GARGARELLA, Roberto. «El nuevo constitucionalismo dialógico frente al sistema de los frenos y los contrapesos». Revista Argentina de Teoría Jurídica, 2013: 1-32. Disponible en: $<$ https://goo.gl/g6gvl>. Consultado en línea febrero 17 de 2017.

GAVILANES, Raul Velásquez. «Hacia una nueva definición del concepto "política pública”». Desafíos 20 (2010): 149-187.

HOLMES, Stephen. «El precompromiso y la paradoja de la democracia». En Constitucionalismo y democracia, de Jon Elster y Rune Slagstad, 217-262. México D.F.: Fondo de Cultura Económica, 1999.

LASSWELL, Harold D. «The decision process: seven categories of functional analysis». Bureau of Governmental Research, College of Business and Public Administration, University of Maryland, 1956.

LINDBLOM, Charles E. «The science of “muddling through"». Public administration review, 1959: 79-88.

MAYNTZ, Renate. «Nuevos Desafíos de la teoría del governance». Instituciones y Desarrollo, 2000: 35-52. 
MCCOMBS, Maxwell, y EVATT, Dixie. «Los temas y los aspectos: explorando una nueva dimensión de la agenda setting». Comunicación y sociedad, 8, nº 1 (1995): 1-20.

NASER, Alejandra, y ALUJAS, Álvaro Ramírez. Plan de gobierno abierto: una hoja de ruta para los gobiernos de la región. Santiago de Chile: CEPAL, 2014.

NINO, Carlos. La Constitución de la democracia deliberativa. Barcelona: Gedisa, 1997.

PORTA, Francesc Pallarés. «Las políticas públicas: El sistema político en acción». Revista de estudios políticos, 1988: 141-162.

POST, Robert, y SIEGEL, Reva. Constitucionalismo Democrático. Por una reconciliación entre Constitución y pueblo. Buenos Aires: Siglo XXI Editores, 2013.

PRATS, Joan. «Políticas de buena administración para la Administración General del Estado en España. Un enfoque de Gobernanza Democrática». En Gobernanza democrática y fiscalidad, 2555. España: Tecnos, 2010.

ROUSSEAU, Jean-Jacques. «El contrato social». En Escritos de combate, de Jean-Jacques Rousseau, 393-526. Madrid: Alfaguara, 1979.

SHAFRITZ, J. M.; RUSSELL, E. W.; \& BORICK, C. Introducing public administration. New York: Routledge, 2015.

SUBIRATS, Joan. «El análisis de las políticas públicas». Gaceta sanitaria 15, nº 3 (2001): 259-264.

VALENCIA AGUDELO, Germán. «Incidencia de la sociedad civil en el ciclo de las políticas públicas». Papel Político 17, n. 2 (2012): 469-496.

Natalia Soledad Aprile Abogada, con orientación en Derecho Público de la Universidad de Buenos Aires (Argentina). Máster en Democracia y Buen Gobierno de la Universidad de Salamanca (España). Profesora de carrera de la Facultad de Jurisprudencia de la Universidad del Rosario. E-mail: natalia.aprile@urosario.edu.co

Mateo Gómez Vásquez Abogado de la Pontificia Universidad Javeriana (Colombia). Máster en Democracia y Buen Gobierno de la Universidad de Salamanca (España). Profesor universitario. E-mail: mateo.gomezv@gmail.com 